This document is a manuscript version of the following article:

Title: Scheduling reefer mechanics at container terminals

By: Hartmann, Sönke

Published in: Transportation Research Part E: Logistics and Transportation Review

Volume 51, May 2013, Pages 17-27

Publisher: Elsevier | Year: 2013

DOI: 10.1016/j.tre.2012.12.007

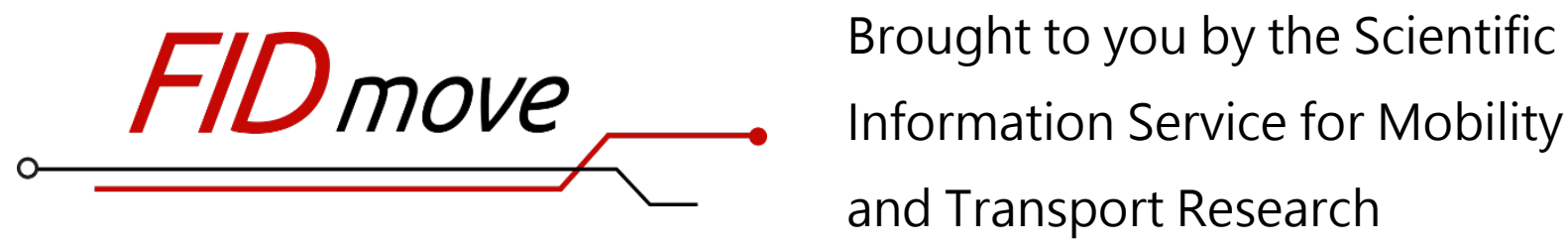

Fachinformationsdienst Mobilitäts- und Verkehrsforschung

Website: www.fid-move.de

Repository: publish.fid-move.de

Contact: publish@fid-move.de

(C) 2013. This manuscript version is made available under the CC-BYNC-ND 4.0 license.

http://creativecommons.org/licenses/by-nc-nd/4.0/ 


\title{
Scheduling Reefer Mechanics at Container Terminals
}

\author{
Sönke Hartmann* \\ HSBA Hamburg School of Business Administration, Alter Wall 38, D-20457 Hamburg, Ger- \\ many. E-mail: soenke.hartmann@hsba.de \\ * supported by the HSBA Foundation
}

\begin{abstract}
This paper discusses the scheduling of reefer mechanics at container terminals. Reefer mechanics plug and unplug reefer containers such that due times are met. We outline the resulting scheduling problem and two simple heuristics. Subsequently, we present a simulation model to analyze the scheduling methods and the reefer-related processes in a realistic dynamic framework. Some results from the simulation experiments are also presented. They demonstrate the applicability of the heuristic and the use of the simulation model in practice. The simulation study was carried out for a real container terminal in the port of Hamburg, Germany.
\end{abstract}

Keywords. Container terminal, reefer, scheduling, heuristic, simulation, case study.

\section{Introduction}

Container terminals are highly complex logistics systems. In order to achieve the best possible performance, optimization and simulation approaches are widely employed in practice. Optimization methods are usually applied to assign tasks to resources such as handling equipment, manpower or storage space. Since there are various terminal designs with different types of handling equipment, different layouts and different processes, many different optimization problems can be found on container terminals. Simulation has been a common tool in the industry since the late 1990s. It is used to analyze processes and optimization methods in a realistic dynamic environment.

Also researchers have devoted their attention to OR methods for container terminals. While practitioners rarely publish their ideas and results, many papers have been published by researchers from universities, especially during the last decade. Excellent literature surveys have been provided by Steenken et al. [28] and Stahlbock and Voß [26] who summarize and classify hundreds of papers. In what follows, we give only a very brief snapshot which may illustrate the broad variety of applications of optimization and simulation to container terminals.

Quay crane scheduling problems are tackled by Peterkofsky and Daganzo [19] and Meisel [16]. Briskorn et al. [5] examine the assignment of transport jobs to automated guided vehicles (AGVs). Gawrilow et al. [10] propose advanced methods for routing AGVs, while Kim et al. [15] discuss deadlock prevention for AGVs. The assignment of transport jobs to straddle carriers is studied in Steenken et al. [27]. Also different types of stacking cranes have been considered. Dekker et al. [6] deal with stacking rules for terminals with automated rail-mounted gantry cranes (RMGs). Speer et al. [25] present an algorithm for scheduling transportation jobs of RMGs. Saanen and 
Valkengoed [22] provide a simulation study in which different RMG designs are compared. Rubbertyred gantry cranes (RTGs) are considered by Zhang et al. [31] who examine the RTG assignment to yard blocks. Zhang et al. [32] discuss the allocation of space for stacking containers in an RTG yard. A unified problem formulation that covers the scheduling of AGVs, RMGs, straddle carriers and manpower is suggested by Hartmann [12]. Hartmann et al. [14] propose a simulation model to analyze the capacity of a quay wall. Going beyond the usual single-terminal applications, Ottjes et al. [17] develop a multi-terminal simulation model. Gambardella et al. [9] suggest to employ a simulation model to support space and resource allocation decisions of the terminal management. Petering [18] employs a simulation model of a transshipment terminal (i.e., a terminal without truck or rail handling) to study the impact of stacking capacity, number of yard canes, number of yard trucks, speed of yard trucks and other factors on quay crane productivity. Hartmann [13] provides a generator of detailed workload scenarios that can be used as input data for simulation models of container terminals. Saanen [21] and Schütt [23, 24] emphasize the importance of emulation. This particular application of simulation allows to test the real terminal operating system (TOS) using realistic equipment simulators.

In this paper, we focus on the scheduling of reefer mechanics on container terminals. Reefer containers are special temperature-controlled containers which are used to transport temperaturesensitive goods such as fresh fruits and vegetables. They are equipped with an integral refrigeration unit and require external power supply while on a ship, truck or train and while being stacked on a container terminal. Reefers can remain without power for a while (up to a few hours), depending on target temperature, outside temperature and sensitivity of the goods. Therefore, they are left without power during a short time interval that is needed for discharging and loading. Reefer mechanics work in the stacking area of a container terminal. They are responsible for unplugging departing containers and for plugging arriving containers. These jobs have to be scheduled such that they are finished before a given due time.

The problem of scheduling reefer mechanics occurs at every container terminal, but it has not yet gained much attention in the scientific literature. To the best of our knowledge, it has only briefly been mentioned by Hartmann [12]. While possibly less crucial than than the scheduling of automated cranes and vehicles, the scheduling of reefer mechanics is of considerable importance to the container terminal management. Containers must be plugged and unplugged on time with a limited workforce or, in other words, at low costs.

This paper presents a case study from a real terminal, the HHLA Container Terminal Altenwerder in Hamburg, Germany. The main purpose of the paper is to give a case study on the integrated application of simulation and optimization in practice. We describe the conditions that are relevant for scheduling the reefer tasks and outline the resulting optimization problem. Then we discuss two simple scheduling heuristics and, subsequently, a simulation model in which the heuristics are tested. Finally, we summarize the results of the simulation. It must be mentioned that, for reasons of confidentiality, some details concerning real values and terminal processes cannot be revealed here. Nevertheless, this paper should give insight into the optimization and simulation approach.

\section{Scheduling Environment}

\subsection{The Container Terminal}

In this paper, we consider the HHLA Container Terminal Altenwerder (CTA) in the port of Hamburg, Germany. Its quay wall with a length of $1400 \mathrm{~m}$ is equipped with double-trolley quay cranes. The quay cranes are responsible for the loading and unloading of the container vessels. Automated guided vehicles transport containers between the quay cranes and the stacking area. The stacking area is divided into yard blocks (sometimes also called modules or substacks). Each of these blocks is served by two automated stacking cranes (rail-mounted gantry cranes, RMGs). On the landside of the terminal, external trucks are served. Internal trucks are employed for the transportation 


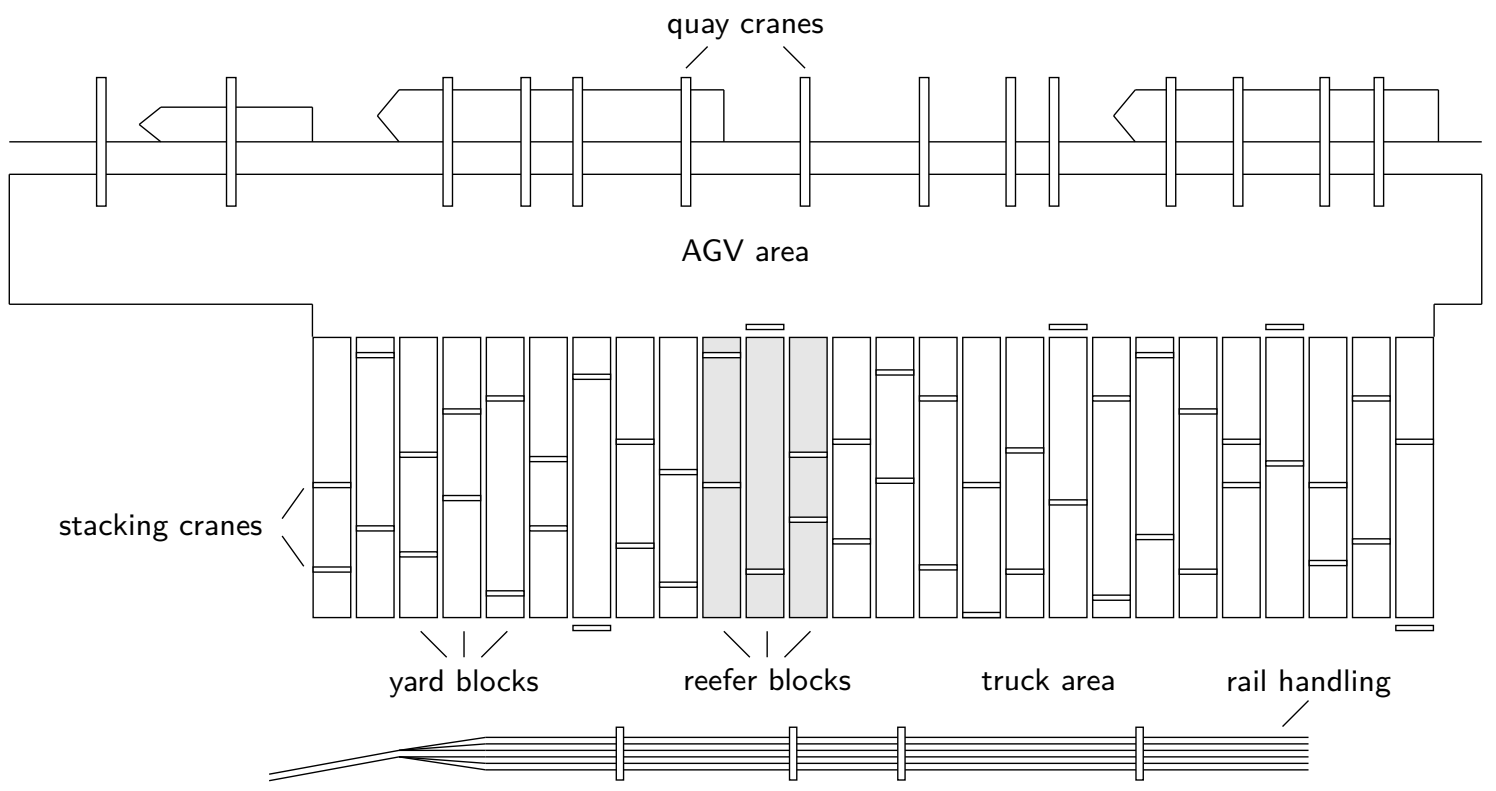

Figure 1: Layout of the container terminal

between the rail area and the stack. The terminal's handling capacity is approximately 3 million TEU (twenty-foot equivalent units, 20' standard containers) per year. The layout of the terminal is sketched out in Figure 1.

The processes on the waterside are as follows: During discharging, a quay crane picks a container on the vessel and puts it onto an AGV. The AGV drives the container to the stack and waits until a stacking crane has picked the container. Then the stacking crane puts the container into the stack. The loading process is vice versa, with the exception that a container to be loaded onto a vessel might cause shuffle moves, that is, a stacking crane might first have to remove containers standing on top of the required container. Then the stacking crane puts the container to be loading onto an AGV which brings it to the quay crane.

The processes on the landside involve both external trucks and rail operations. For deliveries, external trucks drive directly to a block where a stacking crane picks a container and puts it into the stack. For pick-ups, the stacking crane delivers a container from the stack to the truck. Rail unloading is done as follows. A rail crane picks a container from a rail car and puts it onto an internal truck which brings it to the stack where it is taken over by a stacking crane. The process of train loading is analogous. Of course, also container departures on the landside may cause shuffle moves.

All processes are controlled by a software called terminal operating system (TOS). The TOS determines positions in the stack for arriving containers, and it assigns resources such as AGVs, stacking cranes and reefer mechanics to containers.

A few papers on this terminal have been published in the scientific literature. Briskorn et al. [5] and Gawrilow et al. [10] deal with the AGV system while Speer et al. [25] tackle the stacking cranes.

\section{$2.2 \quad$ Reefer Handling}

Parts of the stacking area are equipped with electricity for reefer containers. For this study, it is assumed that three blocks in the center of the stack are reefer blocks, cf. Figure 1 (for reasons of confidentiality, we have employed this design here which differs a bit from that of the real terminal).

The reefer blocks are equipped with racks which enable the reefer mechanics to plug and unplug 
also the containers on the higher levels. To get to the next container, the mechanics have small cars such that they can drive longer distances alongside the blocks. Within the rows of a block, they walk and, to get to the upper levels, climb stairs. The terminal management determines the number of reefer mechanics depending on workload estimates during time intervals. The basic process related to reefer mechanic job handling is as follows:

- The TOS transmits a job (i.e., information on container and location) to a reefer mechanic.

- The reefer mechanic receives the job on his portable radio data device.

- He (or she) drives and/or walks to the specified location.

- He plugs or unplugs the container.

- He confirms the completion of the job.

- The TOS executes the scheduling method and transmits the next job to the mechanic.

The scheduling method is triggered by two types of events. The first one is the completion of a job by a reefer mechanic (see the process above). The second type of event occurs if a new job becomes executable and at least one mechanic is currently idle.

Jobs to plug and unplug reefers are created continuously over time, as trucks and trains arrive and the work on vessels progresses. Each arriving reefer container is related to a plug job, and each departing reefer is related to an unplug job. Shuffling a reefer is associated with both an unplug and a plug job. Each of these jobs has a release time (earliest possible start time) and a due time (latest possible finish time).

The release time of a plug job equals the time the container has been dropped by the stacking crane. The due time of a plug job depends on the allowed time without electricity (usually a buffer is taken into account such that slightly exceeding the due time is not a severe problem). This typically leads to time windows of around one hour for plug jobs. A very few containers with highly sensitive cargo require extremely short time windows.

Considering unplug jobs, the due time is the time at which the stacking crane has to pick the container, possibly minus a buffer time. The release time is derived from the time the job becomes known and the allowed time without power. The length of the time window here depends on the transport mode and type of process. The time window of a reefer going to a vessel or a train is long whereas the time window of a container going to a truck is short. This is because the processes for vessels and trains can be planned for some time in advance while trucks arrive at the terminal gate without prior notice. Time windows of shuffles are generally shorter than those of the related departing container.

The travel times and job durations can be estimated, but the actual times usually fluctuate around these estimates. A small percentage of the jobs take much longer than expected because of unforeseeable mechanical problems when plugging or unplugging. These problems might even require light repair tasks by the reefer mechanic, leading to a long and unpredictable job duration.

\section{Reefer Mechanic Scheduling}

\subsection{Problem Description}

We consider the scheduling problem that arises when an event such as the completion of a job triggers the calculation of a new schedule. The scheduling then involves all currently known jobs which have not yet started and which can be executed now (i.e., the release time is smaller than or equal to the time at which the scheduling method is executed). It also considers all available reefer mechanics and their current states (idle or busy). Taking this into account, the reefer mechanic scheduling problem can be summarized as follows. 
We have $n$ jobs which can be started at any time. Preemption is not allowed, that is, once started, a job must be completed. Each job $j$ is associated with a due time $d_{j}$ by which it should be finished and a processing time $p_{j}$ reflecting the plugging or unplugging. Note that, since all jobs can be started right away, the release time is not needed for the problem formulation (the release time is only used by the system to filter the jobs for scheduling). The travel time from the container related to job $i$ to the container related to job $j$ is referred to by $e_{i j}$. The finish times $f_{i}$ and $f_{j}$ of two consecutive jobs $i, j$ of the same mechanic must satisfy the constraint $f_{i}+e_{i j}+p_{j} \leq f_{j}$. We assume that an estimate of $e_{i j}$ based on distance and speed is given for all jobs $i, j$.

Furthermore, we have $m$ reefer mechanics. Each reefer mechanic $k$ can carry out any job $j$ but only one at a time. Reefer mechanic $k$ has an availability time $a_{k}$ which is the earliest time at which the travel time to his next job may start. The latter is necessary because of the online nature of the process: While at least one mechanic is idle when scheduling is executed, usually several others will still be busy with their current job. Thus the availability time corresponds either to the actual time (i.e., "now") if the mechanic is currently idle or to the estimated completion time of his current job.

A schedule consists of an assignment of each job to a mechanic and a sequence of the jobs of each mechanic, which leads to a planned finish time $f_{j}$ for each job $j$. The objective is to find a schedule with minimum weighted sum of total tardiness and total travel time, that is, we minimize

$$
\omega_{1} \sum_{j=1}^{n} \max \left\{f_{j}-d_{j}, 0\right\}+\omega_{2} \sum_{i, j=1}^{n} e_{i j} x_{i j},
$$

where $x_{i j}=1$ if job $j$ is carried out immediately after job $i$ by the same mechanic and $x_{i j}=0$ otherwise. $\omega_{1}$ and $\omega_{2}$ are the weights of tardiness and travel time, respectively. Objectives of this type are quite common in container terminal logistics, see, e.g., Speer et al. [25] who employ a similar objective for scheduling stacking cranes.

Note that tardiness cannot always be avoided as some new jobs may have very tight due times and all mechanics are still busy. Moreover, the workload is not constant, and it may happen that it temporarily exceeds the capacity of the mechanics. While tardiness is the more important part of the objective, the empty time part is needed especially for times of low workload. During these times, most due times are not tight but schedules with short travel times are needed, especially for the contentment of the mechanics. It should also be mentioned that the goal of travel time minimization implicitly maximizes the productivity of the mechanics. Note that both parts of the objective are not necessarily conflictive as short travel times support the minimization of tardiness.

\subsection{Relationships to Other Optimization Problems}

The reefer mechanic scheduling problem as outlined above is similar to some well-known standard OR problems from the literature. In particular, it is strongly related to machine scheduling (for a survey of machine scheduling problems see Allahverdia et al. [1]). Obviously, the reefer mechanics correspond to the machines and the travel times correspond to setup times. We obtain a machine scheduling problem with multiple identical machines, non-batch sequence-dependent setup times and an objective containing total setup time and total tardiness.

Moreover, there are also some similarities to the multiple traveling salesman problem with time windows, an extension of the well-known traveling salesman problem (TSP), see Bektas [4] for an overview. Clearly, each reefer mechanic corresponds to a salesman. Without loss of generality, the processing times could be included into the travel times. Unlike the multiple TSP, however, there is no depot from which all salesmen start and to which they must return. That is, we have an open multiple TSP with time windows.

Also recall that the reefer mechanic scheduling problem is embedded into a dynamic environment and hence an online optimization problem. Online versions of the machine scheduling problem and the TSP have been discribed by Grötschel et al. [11]. 
In addition, also non-standard problems with similarities to reefer mechanic scheduling can be found in the literature. In particular, field technician scheduling problems have a similar structure. There, technicians have to carry out repair, maintenance and other jobs at different locations such that travel times between locations as well as service times are taken into account. Unlike reefer mechanic scheduling which has a rolling horizon of about one hour, however, usually full days are scheduled, and technicians start at a depot in the morning and have to return in the evening.

$\mathrm{Xu}$ and Chiu [30] deal with technician scheduling in the telecommunication industry. Whereas reefer mechanic scheduling applies due times and a tardiness-based objective, the time windows are constraints here while the objective is to maximize the number of jobs that are actually carried out. A second part of the objective is similar to the minimization of the travel times in reefer mechanic scheduling. Tang et al. [29] consider the maintenance of geographically distributed equipment. Similar to the reefer mechanic scheduling problem, this problem setting includes an objective that captures the urgency of the tasks. A difference is, however, that a separate problem can be defined for each technician because of separate geographic territories. Thus the problem contains only a single technician.

\section{Heuristics}

\subsection{Conditions and Requirements}

The practical situation imposes a few requirements on the optimization methods. First and most important, very short run times (below one second) are necessary. Note that, since the scheduling method will start its calculation of a worker's next job when he has confirmed completion of his last one, the calculation time is idle time for the workers.

It has to be taken into account that up to 100 jobs have to be scheduled in times of high workload (maybe even more if the terminal is expanded). Together with the requirement of short calculation times, this implies that heuristics are more appropriate than exact optimization methods. It should also be mentioned that the often uncertain and changing information that was discussed in Section 2.2 would limit the benefits of optimal schedules anyway.

As mentioned above, we are dealing with an online optimization problem with new jobs arriving all the time. Since the completion of a job triggers the execution of the scheduling method, typically only one job from the last schedule has finished when the scheduling method is called again. Thus one might think of a reoptimization approach which adapts the last schedule by incorporating the new jobs into the last schedule. Reoptimization of the previous solution is usually done when the instance has changed only slightly (see, e.g., Ausiello et al. [2]). Here, however, the instance often changes drastically. This occurs if a large number of new jobs has been created (which happens if a new work package is released by the TOS) or if there have been substantial delays of the current jobs (which also happens from time to time). For these reasons, reoptimization is not considered to be appropriate here. Hence, whenever the scheduling method is executed, the last schedule is discarded and a new one is calculated from scratch, based on the updated information.

\subsection{Dispatching Approach}

The first optimization method is a very simple dispatching heuristic. Given the jobs that are currently known and the current status of the reefer mechanics, we proceed as follows: In a first step, the most urgent job is selected (i.e., the one with the tightest due time). Then, in a second step, it is considered to add this job at the end of each mechanics' current job sequence. The mechanic that leads to the smallest increase in the objective function (weighted additional tardiness and travel time) is chosen, and the job is added at the end of this mechanic's partial schedule. These two steps are repeated until each currently idle mechanic has received a job.

Note that adding more than one job to a mechanic's partial schedule would be unnecessary. The dispatching approach as described above approach would add further jobs at the end of the 
partial schedule, that is, after previously added jobs. Recall, however, that only the first job can be executed from the calculated schedule because rescheduling is initiated when the first job is finished.

The time complexity of the dispatching approach is $O(\mathrm{~nm})$, because a job can be added to each of the $m$ mechanics' partial schedules, and at most $n$ jobs are scheduled until the procedure terminates.

\subsection{Insertion-Based Scheduling Approach}

The second heuristic is a bit more advanced than the first one although still simple and sufficiently fast. It can be seen as a variant of the well-known insertion concept for the TSP, see Rosenkrantz et al. [20].

Again, in the first step, the most urgent job is determined. In the second step, every possible position in the current partial schedule is evaluated. That is, the job is temporarily inserted in every position before and after any previously scheduled job of any mechanic, and the resulting objective function value is calculated. Afterwards, the job is scheduled in the position with the lowest objective function value. The steps are repeated until all jobs have been scheduled.

Observe that, in contrast to the dispatching method, this approach schedules all jobs and not just one for each mechanic. This is because a less urgent job which is considered in a later iteration may be inserted before more urgent jobs and thus might in fact become the first job of a mechanic and thus the job to be executed next.

The rationale behind this approach is as follows. The emphasis is laid on the tardiness minimization and hence on the most urgent jobs in terms of the due time. But as long as due times are not (or only very slightly) exceeded, it is beneficial to insert less urgent jobs whenever they are close to a mechanic's current position. This leads to smaller travel times, which also make it more likely that there is sufficient time to complete future jobs within their time windows. Also note that, when inserting less urgent jobs, it makes sense to build schedules with a longer horizon even though only the first job of a mechanic is executed: We can make the decision to start a job that is not urgent only if we see what the impact on other, more urgent jobs is.

The time complexity of the insertion approach is $O(n(n+m))=O\left(n^{2}+n m\right)$, since $n$ jobs are scheduled and each job can be inserted before any job that has been scheduled in a previous iteration, and it can be added at the end of each of the $m$ mechanics' partial schedules (hence there are up to $n+m$ possible positions for each job). The resulting calculation times were generally short enough. However, if a very large number of jobs is available during a period of time, one might simply limit the number of jobs for scheduling to, say, $n_{\max }$. Then only the $n_{\max }$ most urgent of the $n$ jobs could be scheduled, which limits the calculation time in a straightforward way.

\section{Simulation Model}

\subsection{Overall Structure}

In order to analyze the reefer processes in general and the scheduling of the mechanics in particular, a simulation study was carried out. We employed discrete event-based simulation (see Fishman [7]) and developed a simulation model using emPlant/Plant Simulation, see Bangsow [3] (previously Simple++, see Föhrenbach and Günther [8]). All algorithms within the simulation model were coded in SimTalk.

The structure of the simulation model is shown in Figure 2. The model captures almost the entire terminal, with the reefer part being the most important one for this case study. It consists of two main parts, the material flow and the information flow, and two further components reflecting the input and the output of the model, respectively.

The material flow part consists of the physical resources and the flow of containers between them. The resources are the quay cranes, the AGVs, the stacking cranes and the reefer mechanics 


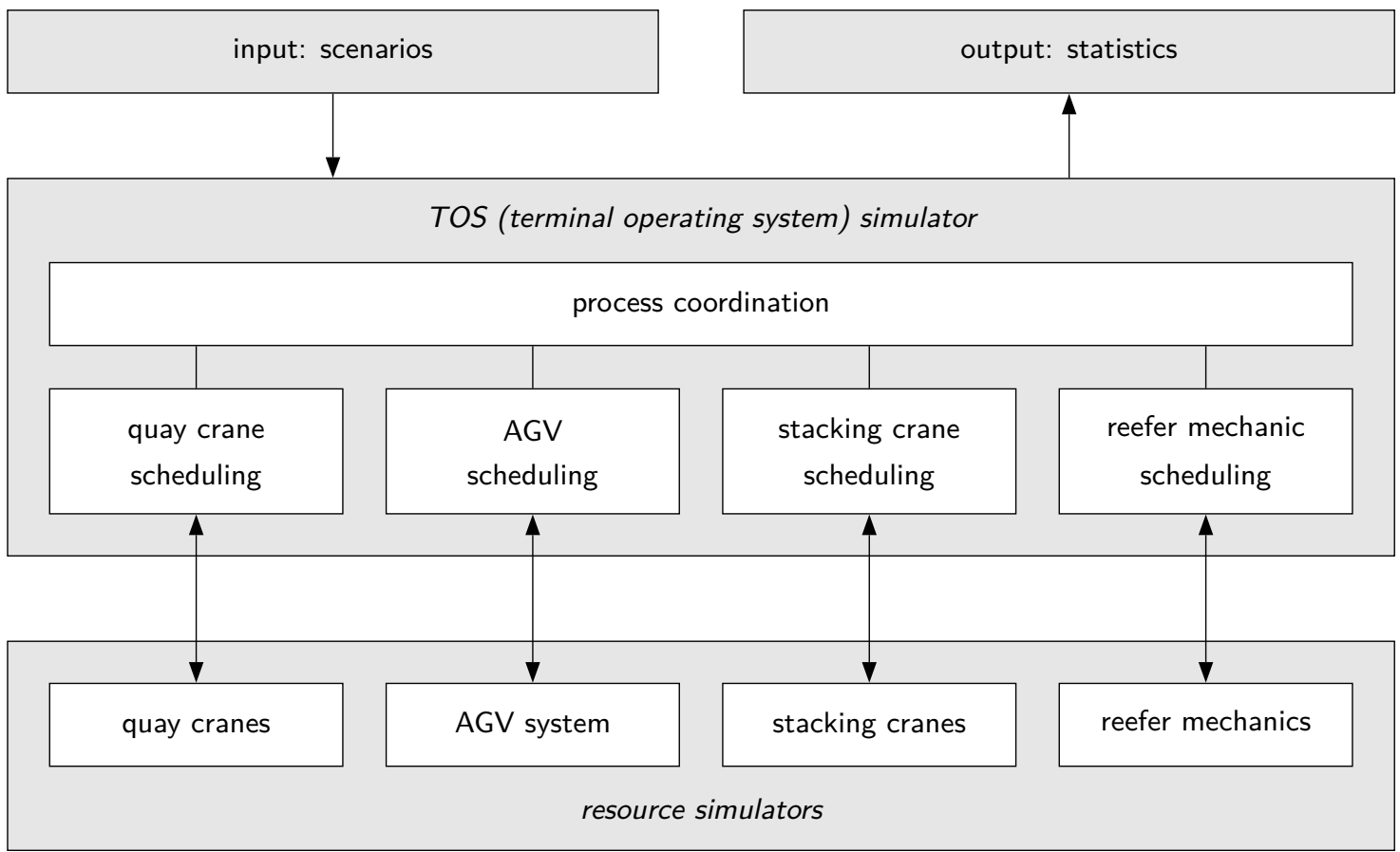

Figure 2: Structure of the simulation model

(the rail cranes were considered to be of less importance, hence they were not included). The information flow part consists of the terminal operating system (TOS) and the flow of messages between the TOS and the resources.

The input information consists of various parameters such as workload and number of reefer mechanics which allow the user to control the scenarios to be simulated. The statistics component collects information from the material and information flow components and determines productivities of the resources, delays, travel and waiting times etc.

The workload of the simulation is controlled as follows: On the waterside, the number of active quay cranes determines the number of containers to be handled and hence the workload. If more quay cranes are active, more containers have to be handled on the terminal. As on the real terminal, each quay crane can work at its maximum productivity defined by the technical specification. The actual productivity, however, is usually lower since it depends on the capabilities of the AGV system, the stacking cranes and also the reefer mechanics (if, for example, a reefer mechanic is late, a quay crane may have to wait for a container to be loaded, which decreases the productivity). On the landside, the number of external and internal trucks arriving at the stacking cranes per hour determines the workload. By selecting the number of active quay cranes and the truck arrival rate, the user can specify any desired terminal workload. Considering reefer handling, the workload also depends also on the number of reefer containers to be handled. Of course, also the capacity of the reefer mechanic workforce is a critical factor. Therefore, a scenario is defined by the user by adjusting the following main parameters:

- Waterside workload: number of active quay cranes

- Landside workload: number of internal and external trucks arriving per hour

- Reefer workload: percentage of reefer containers among all containers

- Reefer capacity: number of available reefer mechanics 
There are many further parameters that can be specified by the user such as number of AGVs and various others (some will be mentioned in the following subsections). Since most of them are not really important for this study, we do not consider them here. For the simulation runs of this study, they have been set to standard values.

\subsection{Material Flow}

The material flow covers the physical resources of the terminal waterside and the stack. The quay cranes are modeled on a detailed level, in particular the two trolleys and the lashing platform. The model does not explicitly allow the quay cranes to move from one ship bay to another. This leads to an even workload throughout the simulation time because there are no bay changes during which a quay crane does not work and hence does not load or unload containers for some time. The AGV system consists of the layout (lanes, curves, crossroads, handover positions) and the vehicles themselves. Whereas major parts are modeled in a realistic way, the claiming mechanism for collision avoidance and the routing that determines the path to the next container pick-up or drop-off location have been simplified in the simulation. The latter was necessary to reduce both the implementation time and the calculation times for the simulation runs. But the impact of the simplification on the behavior, especially concerning the reefer handling which is in the focus of this study, is neglectable. The stacking cranes are captured with considerable detail. Also the interference of the two cranes of a yard block (i.e., the time that one crane may have to wait for the other) is included.

The reefer handling system is modeled at a high level of detail. All physical aspects are included, namely the stairs and platforms, the roads alongside the reefer blocks, the vehicles used by the reefer mechanics and of course the mechanics themselves. In order to be able to analyze potential bottlenecks, special attention has been paid to the limited parking positions at the platforms and to the roads. The latter are, with the exception of certain positions, wide enough for one vehicle only. Also the stochastic variations of the times needed by the reefer mechanics is included. In particular, the model includes parameters that allow the user to specify the probability that an unexpected repair is required as well as the resulting increase of the duration of the job.

For all of the material flow components listed above, realistic values were used. Dimensions of the layout were taken from the original constructional drawings, and dimensions and speeds of the equipment were obtained from the technical specifications. Occasionally, values had to be estimated, especially for the reefer mechanic's speed of driving, walking and climbing stairs. In these cases, realistic estimates were obtained from terminal experts.

\subsection{Information Flow}

In addition to the physical resources, the terminal operating system (TOS) is part of the simulation model. We have implemented a TOS simulator which is a simplified version of the real TOS. Despite some simplifications, the relevant processes and calculations within the simulated TOS are fairly detailed and realistic. A rough sketch of the structure of the TOS simulator can be found in Figure 2 .

The TOS is responsible for the coordination of the processes on the terminal. Each container to be handled is associated with a work order, which is divided into jobs by the TOS. For example, a work order of a reefer container to be loaded onto a vessel is broken up into a reefer mechanic job, a stacking crane job, an AGV job and a quay crane job. The TOS coordinates these related jobs as well as resulting jobs for containers that must be shuffled. The coordination also includes the management of delays (for example, if a loading quay crane is delayed for some reason, the upcoming reefer containers must receive a later due time for unplugging). For arriving containers, the TOS determines a yard block and a slot within that block.

Furthermore, the TOS controls the order in which loaded AGVs drive towards a quay crane. The TOS includes an order in which containers are supposed to arrive at a quay crane. Due to delays in the process (e.g., because of congestion), the AGV with the container expected to arrive 
next might be late, and another AGV with another container might be approaching the quay crane first. In such a case, the TOS will adapt the container order and allow the approaching AGV to deliver its container first. In reality, there are certain degrees of freedom in the planned container order and the quay crane operator can also change the container order manually, such that the behavior of the simulation model can be assumed to be realistic.

To handle the individual jobs, the TOS includes a component for each resource type, that is, quay crane, AGV system, double stacking crane, and reefer mechanic workforce. Each of these components is responsible for the administration of the jobs related to the resource type. This involves collecting the jobs, observing whether they can be released, and scheduling them. For the quay cranes, scheduling is trivial since each quay crane simply takes the next container from the ship bay or from the next AGV, respectively. For the other resource types, the TOS decides based on a heuristic method which resource (i.e., which reefer mechanic, which AGV, and which stacking crane) is doing which job next. For the AGV case, an assignment problem has to be solved (see Briskorn et al. [5]) while a scheduling problem arises for the stacking cranes (cf. Speer et al. [25]) and for the reefer mechanics.

Another task of the TOS is the communication management on the entire terminal. There is no direct communication between different types of resources. That is, an AGV will not tell the stacking crane that it has arrived and can be unloaded, and the stacking crane will not communicate with a reefer mechanic. Instead, as in reality, all communication is made via the TOS. All relevant events are modeled in the simulation. For example, the typical process in the case that an AGV arrives with a reefer container at a yard block is roughly as follows:

- The TOS instructs a stacking crane to go to the waterside handover position.

- A loaded AGV arrives at the destination block and tells the TOS that it has arrived.

- The TOS instructs the stacking crane to pick the container from the AGV and to put it down at a given position.

- When the stacking crane has lifted the container from the AGV, it informs the TOS about it.

- The TOS determines the next job for the AGV and instructs the AGV accordingly.

- When the stacking crane has dropped the container, it informs the TOS about it.

- A reefer mechanic completes his last job and informs the TOS about it.

- The TOS calculates a new reefer schedule which contains the newly dropped container.

- The TOS informs the idle reefer mechanic about his next job (note that this needs not be the new container since it has a rather long time window to be plugged).

All tasks, decisions and calculations of the simulated TOS are implemented with considerable detail on the basis of the original specification of the real TOS. One noteworthy exception is the selection of a slot inside a block. For each arriving container, thousands of slots in the stack would have to be evaluated with regard to several criteria, which would take too much time in the simulation. In order to accelerate the simulation runs, the detailed slot calculation has been replaced by a set of probability distributions. The real slot assignment strategy places containers with certain properties in certain areas of the block. The simulated slot assignment strategy uses probability distributions to find a slot for a container with certain properties. The distributions are derived from the real slot assignment strategy and from typical states of yard blocks such as the filling rate and the stacking height. Consider, for example, a container that will be picked up by a vessel. The real slot assignment strategy will usually stack this container in an area close to the waterside. The simulated slot assignment, however, will simply pick a random slot within this area. Whereas in the real TOS this area is dynamic and depends on the actual situation, the simulated TOS uses a fixed area. 
This leads to acceptable computation times for the simulation model and to sufficiently realistic slots. The probability distributions reflect the actual distribution of containers in the stack that results from the original slot calculation strategy. Note that the slot is only needed for determining the pick-up and drop-off positions in the stacking crane jobs as well as the plug and unplug positions in the reefer jobs. Given that the distributions are realistic, realistic jobs are created for the stacking cranes and the reefer mechanics. It should be added that, due to the simplified slot assignment, shuffle moves cannot be derived from the stack. Consequently, for each departing container, a distribution based on its attributes is applied to generate the number of associated shuffles and the related stacking crane and reefer mechanic jobs.

\section{Simulation Results}

\subsection{Experimental Design}

The workload of a container terminal changes over time. On the waterside, it is higher during days with more vessels at the quay. On the landside, it is higher during daytime and on weekdays. Throughout this study, however, a constant high terminal workload case has been used. This is because workload peaks are the most critical situations in which each subsystem of the terminal must work properly. If the system works well during peak times, other times with lower workload will typically not be a problem. Another point is that a simulation with changing workload might not produce appropriate statistics. Productivities, waiting times, delays etc. are often reported in terms of averages, but these averages are often only meaningful if the workload situation is kept constant over time. Otherwise, large delays during busy times could be evened out by very small delays during less busy times, and the result might be a moderate average delay. This way, critical issues might not be detected.

With this in mind, a large terminal workload has been defined as follows: The quay is fully occupied, and all quay cranes are active. On the landside, the rate of internal and external trucks arriving per hour has been set to the typical peak level. Situations like this usually occur several times per week for several hours.

Based on this high terminal workload, two reefer workload levels, "medium" and "high," have been defined using the parameter that controls the percentage of containers which are reefers. The case with high reefer workload may occur if more quay cranes than usual are working on reefer bays simultaneously. For both reefer workload cases, several numbers of reefer mechanics were used. This was done in order to observe how the system behaves when too few mechanics are available (e.g. because a mechanic has become sick and cannot work) and when a sufficient number of mechanics is available. A scenario in this simulation study therefore consists of a reefer workload level and a number of available mechanics (and of course a high terminal workload). Each scenario was tested with the simple dispatching method as well as with the insertion-based heuristic. The simulation time was 8 hours plus an additional warm-up time at the beginning during which no statistical evaluation was done.

To evaluate the performance of a scheduling method for a scenario, several statistical figures were measured. These figures include the two measures used in the objective function as well as further values which are important for the terminal management:

- For the tardiness we measure the percentage of plug and unplug jobs which are tardy and the average tardiness per tardy job.

- The average travel time per job is determined.

- The productivity is measured as number of jobs per mechanic per hour.

- The number of double cycles is the number of times a mechanic receives a job that is on the same platform as his previous job. Double cycles are considered as positive, especially for the mechanics. 


\begin{tabular}{|c|c|c|c|c|c|c|c|c|}
\hline \multirow{3}{*}{$\begin{array}{l}\text { Reefer rate } \\
\text { mechanics } \\
\text { heuristic }\end{array}$} & \multicolumn{4}{|c|}{ medium } & \multicolumn{4}{|c|}{ high } \\
\hline & \multicolumn{2}{|c|}{3} & \multicolumn{2}{|c|}{4} & \multicolumn{2}{|c|}{6} & \multicolumn{2}{|l|}{7} \\
\hline & dispatch. & insert. & dispatch. & insert. & dispatch. & insert. & dispatch. & insert. \\
\hline tardy jobs & $50 \%$ & $42 \%$ & $6 \%$ & $5 \%$ & $5 \%$ & $6 \%$ & $4 \%$ & $3 \%$ \\
\hline average tardiness & 1.00 & 0.40 & 1.00 & 0.51 & 1.00 & 1.00 & 1.00 & 0.89 \\
\hline average travel time & 1.00 & 0.80 & 1.00 & 0.92 & 1.00 & 0.84 & 1.00 & 0.92 \\
\hline productivity & 1.00 & 1.12 & 1.00 & 1.02 & 1.00 & 1.05 & 1.00 & 1.00 \\
\hline double cycles & 1.00 & 1.57 & 1.00 & 1.58 & 1.00 & 1.96 & 1.00 & 1.53 \\
\hline interference time & 1.00 & 0.41 & 1.00 & 0.85 & 1.00 & 0.54 & 1.00 & 0.79 \\
\hline block changes & 1.00 & 0.71 & 1.00 & 0.85 & 1.00 & 0.90 & 1.00 & 0.88 \\
\hline workforce occupation & $98 \%$ & $98 \%$ & $84 \%$ & $83 \%$ & $97 \%$ & $96 \%$ & $86 \%$ & $84 \%$ \\
\hline
\end{tabular}

Table 1: Simulation results for the scheduling methods

- The interference time is the cumulated time that a mechanic has to wait with his car alongside the block because of an oncoming car.

- The number of block changes is the number of times that a mechanic has to go to another yard block for his next job. Block changes are considered as negative because of possible interference with internal and external trucks.

- The occupation of the workforce is calculated as total time the mechanics are occupied with travel times and jobs divided by the available man-hours of the reefer mechanics.

\subsection{Results of the Simulation Runs}

The most important results of the simulation runs are summarized in Table 1. Unfortunately, all absolute values must be kept confidential because this is a simulation study based on real data. Therefore, we can give only relative values. The results of the dispatching method have been used as a basis, and the result of the insertion heuristic for the same reefer workload and the same number of mechanics is given in relation to this basis (the base value is indicated by a factor of 1.00). The table is to be read as follows. Consider, for example, the medium workload case. If three reefer mechanics are available, the productivity achieved by the insertion method is $12 \%$ higher (factor 1.12 in Table 1) than that obtained from the dispatching approach. If four mechanics are available, the productivity related to the insertion procedure is $2 \%$ higher (factor 1.02) than that of the dispatching method.

The results of Table 1 show that the insertion heuristic clearly outperforms the simple dispatching method. For the same scenario, it leads to less tardy jobs (with the exception of the high workload case with 6 reefer mechanics), less average tardiness, less average travel time, higher productivity, more double cycles, less car interference time, and less block changes. As expected, the dispatching method is too simple, and the insertion heuristic seems to be capable of inserting less urgent jobs into the mechanics' job sequences to achieve lower travel times, which increases the productivity and reduces the tardiness.

The superiority is most obvious when the number of mechanics is clearly too small for the actual workload. Such a situation may happen in practice, for example if the workload is higher than expected or if a mechanic becomes sick. Consider especially the scenario with medium reefer workload and only three mechanics, where roughly half of the jobs exceed their due times. In such a critical case, the benefits of the scheduling method are of particular importance. In the simulated scenario, the insertion method was able to handle $12 \%$ more reefer containers per hour than the dispatching approach, and the average tardiness per job was less then half of the tardiness produced by the dispatching method. 
When the capacity of the workforce is scarce, the mechanics are occupied for almost $100 \%$ of their time. The rate is slightly below $100 \%$ because it may happen from time to time that fewer reefer containers have to be handled, such that some mechanic does not receive a next job immediately. When more mechanics are available for the same workload, the occupation drops well below $90 \%$, which indicates that the capacity should be sufficient. Nevertheless, a small percentage of the jobs is still not on time. This due to two reasons: First, there might be many trucks requesting reefers during a short interval. Since these jobs have tight due times and the mechanics have to finish their current jobs first, they cannot carry out the new jobs on time. Second, a few containers require unexpected repair, which increases the job duration drastically. Consequently, the job might finish too late, and the mechanic is unavailable for other jobs during this time. These effects imply that it is usually not possible to carry out every job on time, even with the best possible scheduling method or a few additional mechanics. In fact, the high workload case was additionally tested with 12 mechanics which led to a workforce occupation of only $44 \%$, but still $1 \%$ of the jobs were late.

Since absolute values cannot be given, Table 1 does not show how large the tardiness values and the travel times actually are. Therefore, we give some further remarks that cannot be seen from the relative values in the table. Only in the case of three mechanics and medium workload, the average tardiness is substantial. In the other cases, the average decreases to acceptable levels. It should also be mentioned that the travel times in the high workload scenarios are generally smaller than those in the medium workload cases. This is because more reefer jobs make it more likely that a nearby job can be assigned to a mechanic. Finally, the absolute interference times are rather small such that one can conclude that the roads alongside the blocks are not a bottleneck.

Several additional experiments were carried out using the simulation model. In order to illustrate the practical applications of the model, we summarize some of them.

- Various settings for the weights of the objective function (which is used in the heuristics) have been tested. As long as reasonable settings were considered, the results did not differ much. This indicates that the scheduling approach is robust.

- Further experiments were carried out to analyze parameter $n_{\max }$ which limits the number of jobs for scheduling. The results indicate that if $n_{\max }$ is set to 40 , the computation times are acceptable, and the quality of the scheduling results in terms of tardiness and travel times is only very marginally affected (of course, this result is related to the terminal size and workload under consideration in this study).

- Since block changes are considered to be negative with regard to the terminal processes, they should be reduced as much as possible. Several simulation runs with a penalty for each block change in the objective function were carried out. The results show that appropriate penalty values were able to reduce the number of block changes by almost 50\%. Productivity and tardiness were hardly affected, but this result only holds if the number of mechanics is not too small.

- The temperature of reefer containers have two be checked three times per day. These socalled monitoring jobs are usually carried out by a separate pool of mechanics and were not considered in the above experiments. An additional set of experiments has been conducted to compare the case of one pool for plugging, unplugging and monitoring with the case of two separate pools. The simulation showed that having one pool is more efficient with regard to productivity per person.

\section{Conclusions}

In this paper the scheduling of reefer mechanics on container terminals was discussed. Efficient scheduling of reefer jobs is of particular importance for the terminal management. The study has 
shown that the choice of the scheduling method has a crucial impact on the productivity of the reefer mechanic workforce and on the completion of the jobs before their due times, especially if the capacity of the workforce is scarce. The outcome of this simulation study has been taken into account in practice, that is, the scheduling of reefer mechanics in the real terminal operating system is done as recommended in this study.

Like many other systems in logistics and production, container terminals are characterized by complex, dynamic and uncertain processes. The complexity lies in the interrelation of the different resource types - reefer mechanics, stacking cranes, automated guided vehicles and quay cranes are highly dependent on each other. New jobs arrive all the time which implies a dynamic environment. The uncertainty is due to travel times and jobs which may take much longer than expected. Under these conditions, an optimization method such as a reefer mechanic scheduling heuristic can only be fully evaluated within a simulation model that reflects these conditions in a realistic way.

But it must also be emphasized that a simulation model is more than just a test bed for optimization methods. It can be used for analyzing potential or actual bottlenecks in the material flow. Moreover, it can be employed to test alternative processes which are related to operational issues. All these applications have been discussed in this paper using a case study from a real container terminal. This broad variety of applications makes simulation an indispensable tool in practice.

The contributions of this paper to the literature are twofold. First, it proposes the first tailored method to solve the reefer mechanic scheduling problem which occurs at every container terminal (this problem has only briefly been mentioned by Hartmann [12] before). Second, this paper presents a detailed simulation model for a real-world container terminal. While there are several excellent simulation models for container terminals in the scientific literature (see the references given in the introduction), many of them cover only a subsystem rather than the entire terminal (e.g., the waterside or the stacking area), and most of them are rather academic in nature and do not always take the requirements and processes of real terminals into account.

Acknowledgements. The author would like to thank Andreas Kohtz for his contribution to the implementation, Holger Schütt and the late Dirk Steenken for the inspiring discussions, and Gerlinde John for her support.

\section{References}

[1] A. Allahverdia, C. T. Ng, T. C. E. Cheng, and M. Y. Kovalyov. A survey of scheduling problems with setup times or costs. European Journal of Operational Research, 187:985-1032, 2008.

[2] G. Ausiello, B. Escoffier, J. Monnot, and V. T. Paschos. Reoptimization of minimum and maximum traveling salesmans tours. Discrete Applied Mathematics, 7:453-463, 2009.

[3] S. Bangsow. Manufacturing Simulation with Plant Simulation and Simtalk. Springer, Berlin, Germany, 2010.

[4] T. Bektas. The multiple traveling salesman problem: an overview of formulations and solution procedures. Omega, 34:209-219, 2006.

[5] D. Briskorn, A. Drexl, and S. Hartmann. Inventorybased dispatching of automated guided vehicles. $O R$ Spectrum, 28:611-630, 2006.

[6] R. Dekker, P. Voogd, and E. van Asperen. Advanced methods for container stacking. OR Spectrum, 28: 563-586, 2006
[7] G. S. Fishman, editor. Discrete-event simulation: modeling, programming, and analysis. Springer, Berlin, Germany, 2001.

[8] A. Föhrenbach and H.-O. Günther. Simple++. OR Spectrum, 22:197-202, 2000.

[9] L. M. Gambardella, A. E. Rizzoli, and M. Zaffalon. Simulation and planning of an intermodal container terminal. Simulation, 21:107-116, 1998.

[10] E. Gawrilow, E. Köhler, R. H. Möhring, and B. Stenzel. Dynamic routing of automated guided vehicles in real-time. In H.-J. Krebs and W. Jäger, editors, Mathematics - Key Technology for the Future, pages 165-177. Springer, Berlin, Germany, 2008.

[11] M. Grötschel, S. O. Krumke, J. Rambau, T. Winter, and U. Zimmermann. Combinatorial online optimization in real time. In M. Grötschel, S. O. Krumke, and J. Rambau, editors, Online optimization of large scale systems, pages 679-704. Springer, Berlin, Germany, 2001.

[12] S. Hartmann. A general framework for scheduling 
equipment and manpower at container terminals. OR Spectrum, 26:51-74, 2004.

[13] S. Hartmann. Generating scenarios for simulation and optimization of container terminal logistics. $O R$ Spectrum, 26:171-192, 2004.

[14] S. Hartmann, J. Pohlmann, and A. Schönknecht. Simulation of container ship arrivals and quay occupation. In J. W. Böse, editor, Handbook of Terminal Planning, pages 135-154. Springer, Berlin, Germany, 2011.

[15] K. H. Kim, S. M. Jeon, and K. R. Ryu. Deadlock prevention for automated guided vehicles in automated container terminals. OR Spectrum, 28:659679, 2006.

[16] F. Meisel. The quay crane scheduling problem with time windows. Naval Research Logistics, 58:619636, 2011.

[17] J. A. Ottjes, H. P. M. Veeke, M. B. Duinkerken, J. C. Rijsenbrij, and G. Lodewijks. Simulation of a multiterminal system for container handling. $O R$ Spectrum, 28:447-468, 2006.

[18] M. E. H. Petering. Decision support for yard capacity, fleet composition, truck substitutability, and scalability issues at seaport container terminals. Transportation Research Part E: Logistics and Transportation Review, 47(1):85-103, 2011.

[19] R. I. Peterkofsky and C. F. Daganzo. A branch and bound solution method for the crane scheduling problem. Transportation Research B, 24:159172, 1990 .

[20] D. J. Rosenkrantz, R. E. Stearns, and P. M. Lewis. An analysis of several heuristics for the traveling salesman problem. SIAM Journal on Computing, 6:563-581, 1977.

[21] Y. A. Saanen. Using emulation to improve the performance of your TOS. Port Technology International, 29:77-81, 2007.

[22] Y. A. Saanen and M. V. Valkengoed. Comparison of three automated stacking alternatives by means of simulation. In Proceedings of the 37th Winter Simulation Conference, pages 1567-1576. ACM, 2005.
[23] H. Schütt. Optimising container terminals using simulation and emulation methodology: Part 1. Port Technology International, 41:81-82, 2009.

[24] H. Schütt. Optimising container terminals using simulation and emulation methodology: Part 2. Port Technology International, 42:62-66, 2009.

[25] U. Speer, G. John, and K. Fischer. Scheduling yard cranes considering crane interference. In J. Böse, H. Hu, C. Jahn, X. Shi, R. Stahlbock, and S. Voß, editors, Proceedings of the Computational Logistics Conference, volume 6971 of Lecture Notes in Computer Science, pages 321-340. Springer, Berlin, Germany, 2011.

[26] R. Stahlbock and S. Voß. Operations research at container terminals - a literature update. OR Spectrum, 30:1-52, 2008.

[27] D. Steenken, A. Henning, S. Freigang, and S. Voß. Routing of straddle carriers at a container terminal with the special aspect of internal moves. $O R$ Spectrum, 15:167-172, 1993.

[28] D. Steenken, S. Voß, and R. Stahlbock. Container terminal operations and operations research - a classification and literature review. OR Spectrum, 26:3-49, 2004.

[29] H. Tang, E. Miller-Hooks, and R. Tomastik. Scheduling technicians for planned maintenance of geographically distributed equipment. Transportation Research Part E: Logistics and Transportation Review, 43(5):591-609, 2007.

[30] J. Xu and S. Chiu. Effective heuristic procedures for a field technician scheduling problem. Journal of Heuristics, 7(5):495-509, 2001.

[31] C. Zhang, Y.-W. Wan, J. Liu, and R. J. Linn. Dynamic crane deployment in container storage yards. Transportation Research B, 36:537-555, 2002.

[32] C. Zhang, J. Liu, Y.-W. Wan, K. G. Murty, and R. J. Linn. Storage space allocation in container terminals. Transportation Research B, 37:883-903, 2003. 